\title{
Hepatic epithelioid angiomyolipoma with prominent invasive growth pattern: A hitherto unreported histopathologic feature which might promote misdiagnosis
}

\author{
Chang Zhao ${ }^{1}$, Xiang-yong $\mathrm{Li}^{2}$, Yu-hang Pan $^{1}$, Si-dong Xie ${ }^{3}$, Jing Zhou ${ }^{*}$ and Jian-ning Chen ${ }^{1 *}$ \\ ${ }^{1}$ Department of Pathology, The Third Affiliated Hospital, Sun Yat-sen University, No.600 Tianhe Road, Guangzhou 510630, China \\ ${ }^{2}$ Department of Infectious Diseases, The Third Affiliated Hospital, Sun Yat-sen University, No. 600 Tianhe Road, Guangzhou 510630, China \\ ${ }^{3}$ Department of Radiology, The Third Affiliated Hospital, Sun Yat-sen University, No. 600 Tianhe Road, Guangzhou 510630, China
}

\begin{abstract}
Background: Hepatic angiomyolipoma (AML) is a rare tumor which composes of variable admixtures of adipose tissue, spindle or epithelioid myoid cells and thickwalled blood vessels. Although an invasive growth pattern into the parenchyma at the tumor-non-tumor interface and/or along the vessels was reported to be found in hepatic AML, hepatic AML with prominent invasive growth pattern has not been reported.

Case presentation: We reported a case of a 25 -year-old man with dull pain in his right upper abdomen. A liver space-occupying lesion was detected by ultrasound and magnetic resonance imaging. Laboratory tests showed normal liver function and negative hepatitis B or C markers. Partial hepatectomy was performed. Microscopically, the tumor was composed of little adipose tissue, thick-walled blood vessels and large number of spindled or epithelioid myoid cells. The spindleepithelioid cells had abundant clear or acidophilic cytoplasm and small nucleolus; they diffusely infiltrated the liver parenchyma along the hepatic sinuses without replacing the hepatocytes. Immunostaining analyses revealed that the epithelioid neoplastic cells were strongly and diffusely positive for HMB45 and weakly positive for Melan-A. This lesion was diagnosed as hepatic AML, which showed a prominent invasive growth pattern.
\end{abstract}

Conclusions: To the best of our knowledge, this is the first hepatic AML with prominent invasive growth pattern described. This unusual histopathologic appearance might promote misdiagnosis, thus requiring for proper identification.

\section{Introduction}

Angiomyolipoma (AML) is a benign mesenchymal tumor that is belongs to a group of perivascular epithelioid cell tumors called PEComa [1]. It defined as a tumor which consists of spindle or epithelioid myoid cells, mature fat, and a thick-walled vasculature. These three components were mixed together in different proportions. The spindle or epithelioid myoid cells exhibit myomatous and lipomatous differentiation with melanogenesis [2,3]. Therefore, they show a characteristic expression of both melanocytic (such as HMB45 and Melan-A) and myogenic markers (such as SMA). The expression of S100, desmin and CD117 in myoid cells were variable.

The kidneys and perirenal soft tissues were the most predilection site for AML, followed by the liver [2]. The histopathology of hepatic AML was the same as that in other anatomical sites, except that hepatic AMLs more commonly showed an epithelioid appearance and invasive growth pattern $[3,4]$. Nonomura, et al. reported that an invasive growth pattern into the surrounding hepatic parenchyma, portal triads and/or around hepatic veins was found in 24 of $39(62 \%)$ hepatic AMLs.

Two invasive growth patterns of hepatic AML were described in the literature [4]. Tumor cells invade into the parenchyma at the tumornon-tumor interface was the one of the invasive growth patterns. In this pattern, small isolated tumor cell clusters were occasionally found in the vicinity of the main mass, showing a sprouting or budding appearance. The other one of the invasive growth patterns showed that tumor cells grow into portal triads or around hepatic veins. Although these invasive growth patterns could be found in hepatic AML, most hepatic AML were well-circumscribed. Even though infiltration of parenchyma by tumor cells can be observed, it is only seen at the tumor-non-tumor interface with small amount [4]. By now, tumor cells diffusely infiltrated in liver parenchyma have not been documented.

Here, we described a case of hepatic AML which have a rare growth pattern that tumor cells diffusely infiltrated the liver parenchyma along the hepatic sinusoids. Given that the invasive growth pattern is one of the most important histological features differentiating malignant tumors from benign ones, the case of hepatic AML in the present study might cause difficulties in diagnosis and raise differential diagnosis of a mimicker of malignancy.

*Correspondence to: Jing Zhou, Department of Pathology, The Third Affiliated Hospital, Sun Yat-sen University, No. 600 Tianhe Road, Guangzhou 510630, China, E-mail: Wyzhj99@163.com

Jian-ning Chen, Department of Pathology, The Third Affiliated Hospital, Sun Yat-sen University, No. 600 Tianhe Road, Guangzhou 510630, China, E-mail: chjning@mail.sysu.edu.cn

Key words: angiomyolipoma, liver, invasive growth pattern, histopathology, diagnosis and differential diagnosis

Received: October 29, 2018; Accepted: November 12, 2018; Published: November 15, 2018 
Zhao C (2018) Hepatic epithelioid angiomyolipoma with prominent invasive growth pattern: A hitherto unreported histopathologic feature which might promote misdiagnosis

\section{Case presentation}

A 25-year-old man was admitted in our hospital because of dull pain in his right upper abdomen accompanied by abdominal distension for 2 months. The past medical history revealed that he did not consume alcohol or tobacco. No organomegaly was noted in the abdomen on physical examination. Laboratory tests showed normal liver function and negative hepatitis B or C markers. Carbohydrate antigen (CA) 19-9, CA 125, CA 15-3, carcinoembryonic antigen (CEA), and alphafetoprotein (AFP) levels were not elevated. Magnetic resonance imaging (MRI) displayed a $5.2 \mathrm{~cm} \times 5 \mathrm{~cm}$ mass in Segments $5 / 6$ (S5/6) of the liver with abundant blood supply (Figure 1), which was considered as AML on imaging studies.

The patient underwent partial hepatectomy. On macroscopic inspection, a solitary mass sized about $5 \mathrm{~cm}$ in diameter was found adjacent to the capsule of the liver. The cut surface of the mass was solid, soft, yellow or brown. The mass was well-circumscribed but unencapsulated with indistinct border (Figure 2A).
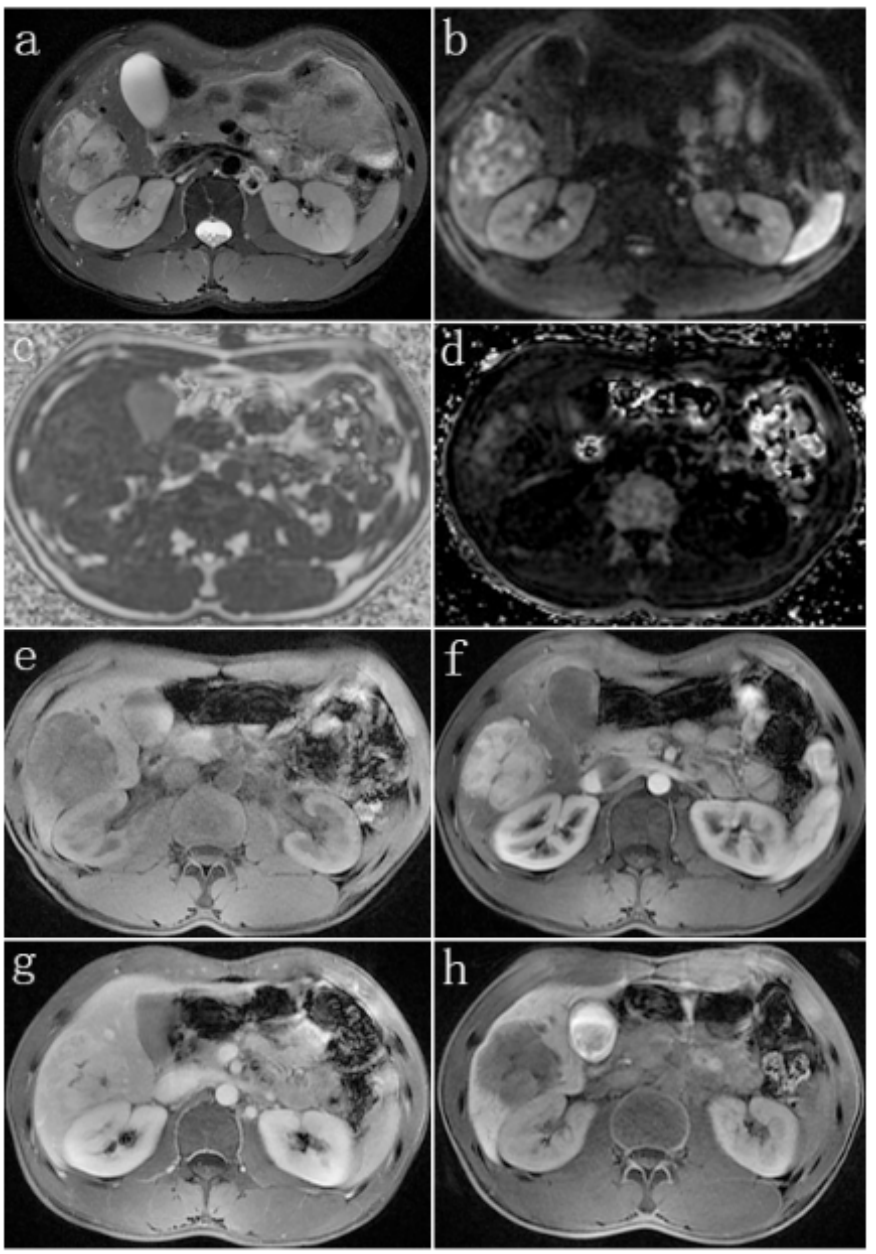

Figure 1. MR images in a 25-year-old man with hepatic AML

A well-defined hepatic mass whose diameter was $5.2 \mathrm{~cm}$ was found in $\mathrm{S} 5 / 6$, with a heterogeneous hyperintensity in T2WI (a). and DWI with a b value of $800 \mathrm{sec} / \mathrm{mm}^{2}$ (b). The quantitative measurement of fat content in the mass was about $8.4 \sim 13.9 \%$ (c). and the $\mathrm{R} 2^{*}$ value of the high signal area in the tumor was estimated to be about $220 \mathrm{~Hz}$, indicating the combination of hemorrhage (d). Multiphasic MR technique with Gd-BOPTA contrast agent showed large hypointense mass in pre-contrast image (e). hyper-enhancement in late hepatic arterial phase (f). persistent enhancement in transitional phase image (g). and finally hypointense relative to enhanced liver parenchyma in hepatobiliary phase obtained at 90 minutes after injection $(\mathrm{h})$.
On microscopic examination, the tumor was composed of little adipose tissue, thick-walled blood vessels and large number of spindled or epithelioid myoid cells (Figure 2B). The spindle-epithelioid cells occupied more than $90 \%$ of the solid area and were found infiltrating along the hepatic sinuses (Figure 2C and 2D). The myoid tumor cells had abundant clear or acidophilic cytoplasm and small nucleolus (Figure 2E). Some tumor cells contained pigment particles (Figure 2F). Mitotic figures were rare. Scattered lymphocytes and plasma cells were found within the tumor (Figure $2 \mathrm{~F}$ ). Focal areas of hemorrhage were present. Extramedullary hematopoiesis and necrosis were absent.

Immunohistochemical staining (Table 1) revealed that the spindleepithelioid neoplastic cells were strongly and diffusely positive for HMB45 (Figure 3A and 3B) and weakly positive for Melan-A (Figure 3C) but were negative for hepatocyte (Hep Par-1), CK, S100 (Figure 3D), ALK (Figure 3E), CD21, CD23, CD68 and TFE3. Vimetin was positive in spindle-epithelioid neoplastic cells (Figure 3F). Adipocytes and blood vessels within the tumor were positive for S-100 and CD34, respectively. Ki-67 index was low ( 3\%).

Besides, double-staining of Hep Par-1 and HMB45 clearly highlighted that the tumor cells grow along the hepatic sinusoids (Figure 3A and 3B); this kind of growth pattern was found nearly in the whole tumor, which showed a prominent invasive growth pattern.

The combined morphologic and immunohistochemical findings suggested a diagnosis of hepatic AML with a prominent invasive growth pattern, which has not previously been reported in hepatic AML. The patient was still alive and remained well in follow-up for 1 year after surgery, without evidence of recurrence or metastasis.

\section{Discussion}

The hepatic AML case we reported here had a more remarkable invasive growth pattern that tumor cells were extensively infiltrated into the liver sinus. To our best knowledge, this kind of growth pattern has not been previously reported in hepatic AML.

Hepatic AML was usually regarded as a benign tumor, however, an invasive growth pattern into surrounding hepatic parenchyma, portal triads and/or around hepatic veins could be found [4]. According to Nonomura, et al's study, two types of invasive growth pattern were identified. In the former pattern, tumor cells replaced hepatocytes within the liver cell cords along the hepatic sinusoids at the tumournon-tumour interface; in the latter pattern, tumor cells grow into the portal triads and/or around the hepatic veins. In both invasive patterns, tumor cells seemed to infiltrate along blood vessels, showing intimate contact with the blood vessel wall.

Although invasive growth could be found in some hepatic AMLs, it does not indicate malignancy. In fact, true malignant AMLs were extremely rare and only several cases have been reported to date [5-9]. These malignant hepatic AMLs were found to be recurred or metastasis and might be died due to the tumor.

By now, there is no consonance of opinion about the criteria of malignant hepatic AMLs. Histological factors predicting the malignant progression of PEComa, such as tumor size $>5 \mathrm{~cm}$, infltrative growth pattern, high nuclear grade, mitosis $>1 / 50 \mathrm{HPF}$, necrosis, and vascular invasion were proposed by Folpe, et al. $[10,11]$. AML is a member of PEComa family, these criteria may also be applicable for hepatic AML. However, criteria differences in different organs may exist. Nese, et al. proposed a risk stratification model to classify renal AMLs into low-, intermediate-, and high-risk categories [12]. The risk factors 
Zhao C (2018) Hepatic epithelioid angiomyolipoma with prominent invasive growth pattern: A hitherto unreported histopathologic feature which might promote misdiagnosis

Table 1. Antibodies used for immunohistochemical staining

\begin{tabular}{|c|c|c|c|}
\hline Antibody & Major Specificity & Source & Dilution \\
\hline \multicolumn{4}{|l|}{ Epithelial marker } \\
\hline Cytokeratin (AE1/AE3) & Pan-cytokeratin & Novocastra & $1: 100$ \\
\hline Cytokeratin 7 (OV-TL12/30) & Bile duct epithelial cells & Dako & $1: 150$ \\
\hline Cytokeratin 19 (b170) & Bile duct epithelial cells & Novocastra & $1: 150$ \\
\hline \multicolumn{4}{|c|}{ Mesenchymally-derived cell marker } \\
\hline Vimentin (V9) & Mesenchymally-derived cell & Dako & 1:1000 \\
\hline Smooth muscle actin ( $\alpha \mathrm{sm}-1)$ & Muscle cells, myofibroblasts, pericytes & Novocastra & $1: 100$ \\
\hline S-100 (antiserum) & $\begin{array}{l}\text { Melanocytes, dendritic cells, nerve sheath } \\
\text { cells, cartilage cells, myoepithelial cells, } \\
\text { adipocytes }\end{array}$ & Dako & $1: 1000$ \\
\hline Melanoma (HMB45) & Melanocytes, myoid cells & Novocastra & $1: 100$ \\
\hline Melan-A (A103) & Melanocytes, myoid cells & Novocastra & $1: 50$ \\
\hline TFE3 (MRQ-37) & Transcription Factor E3 & Dako & RTU \\
\hline ALK (5A4) & Anaplastic Lymphoma Kinase & Novocastra & RTU \\
\hline \multicolumn{4}{|l|}{ Endothelial markers } \\
\hline CD31 (JC/70A) & Endothelial cells, megakaryocytes & Dako & $1: 50$ \\
\hline CD34 (QBEnd/10) & Endothelial cells, some stromal cells & Novocastra & $1: 200$ \\
\hline ERG (EP111) & Endothelial cells & Dako & $1: 50$ \\
\hline \multicolumn{4}{|l|}{ Follicular dendritic cell markers } \\
\hline CD21 (2G9) & Follicular dendritic cells & Novocastra & RTU \\
\hline CD23 (1B12) & Follicular dendritic cells & Novocastra & RTU \\
\hline \multicolumn{4}{|l|}{ Histiocyte and monocyte marker } \\
\hline CD68 (514H12) & Histiocytes and monocytes & Novocastra & $1: 100$ \\
\hline \multicolumn{4}{|l|}{ Proliferation-related marker } \\
\hline Ki-67 (MIB-1) & Cell proliferation activity & Dako & $1: 100$ \\
\hline
\end{tabular}

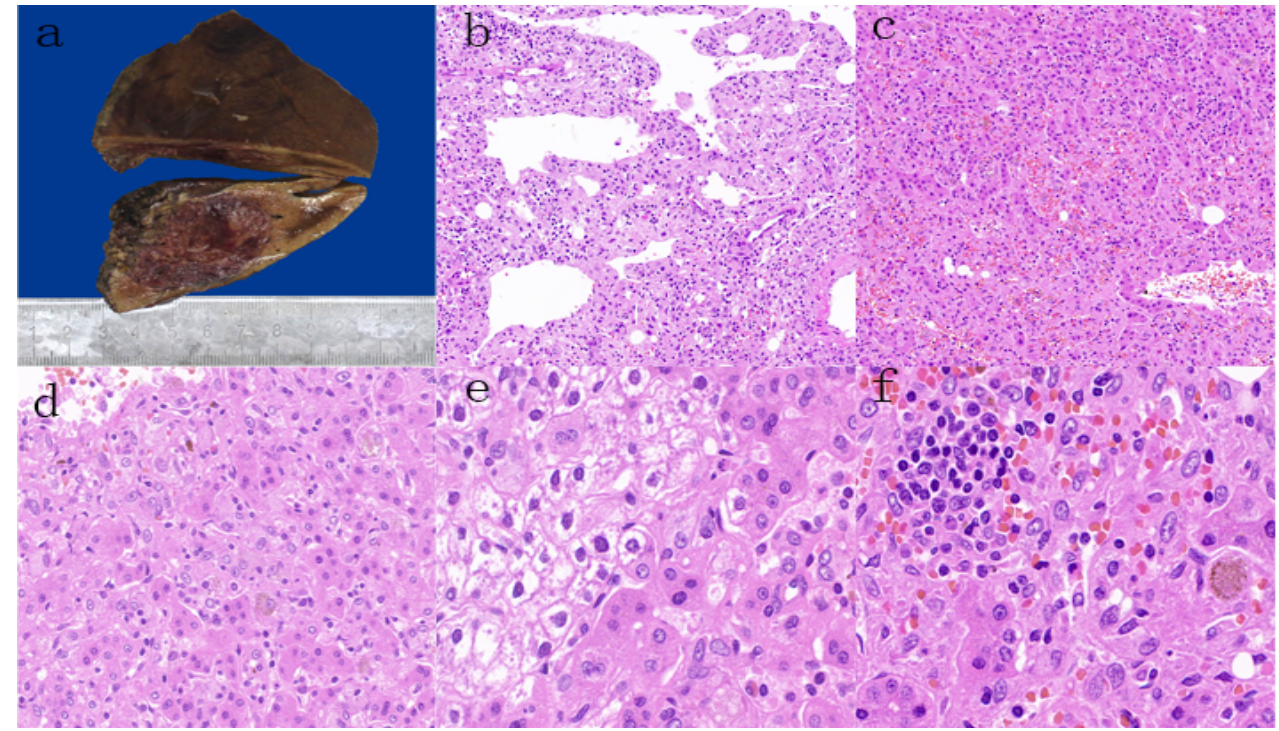

Figure 2. Pathology of the hepatic AML

a. The cut surface of the resected liver illustrated a solitary and dark-red mass with unclear borders and necrosis (10\% buffered formalin-fixed). b. Low-power view showed the three components of the tumor: spindle-epithelioid myoid cells, fat cells and thick-walled blood vessels. Some areas of the tumor arrange in cracks (H\&E, $\times 100)$. c. Epithelioid tumor cells diffusedly infiltrated within the hepatic sinuses with scattered lymphocytes and plasma cells infiltration $(\mathrm{H} \& \mathrm{E}, \times 100)$. d. Sinusoidal infiltration by the epithelioid tumor cells $(\mathrm{H} \& \mathrm{E}, \times 200)$. e. Epithelioid cells had clear or light eosinophilic cytoplasm and small nuclei, which invaded the adjacent normal liver (H\&E, $\times 400)$. f. Aggregates of lymphocytes and plasma cells and scattered pigment particles in tumor cells were found in some areas within the tumor $(\mathrm{H} \& \mathrm{E}, \times 400)$.

included concurrent AML or associated tuberous sclerosis complex, necrosis, tumor size $>7 \mathrm{~cm}$, extrarenal extension and/or renal vein involvement, and a carcinoma-like growth pattern. In hepatic AML, as mentioned above, infltrative growth pattern does not indicate malignancy. In addition, cytologic atypia could be observed both in benign and malignant hepatic AMLs, and thus could not be served as a histopathologic feature in distinguishing benign and malignant AML [5]. In Nguyen, et al's study [5], coagulative necrosis, tumor size $>7 \mathrm{~cm}$, evidence of matastasis or death attributed to the tumor, and absence of CD117 expression in tumor cells suggest malignant hepatic AMLs. None of the features was found in our case, suggesting that this is a benign tumor. However, careful follow-up of the patient is still recommended. 


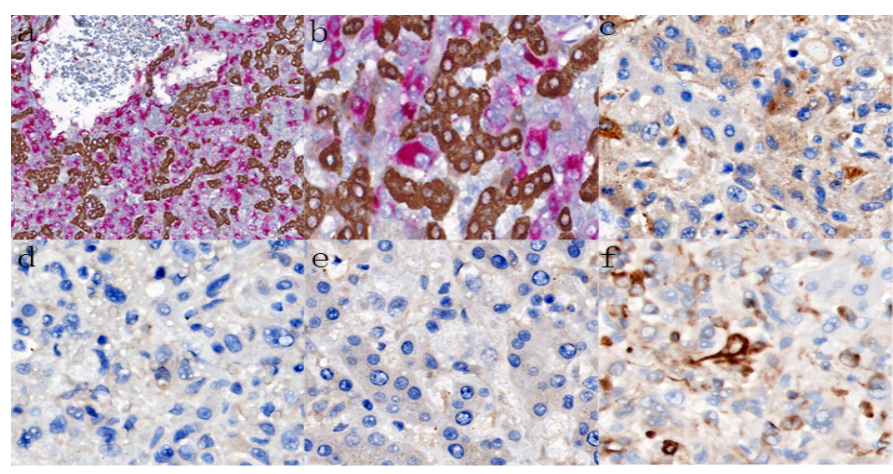

Figure 3. Immunostaining of the hepatic AML

a-b Double immunostaining for hepatocyte (brown) and HMB45 (red). The hepatocytes were stained brown, while the epithelioid tumor cells were stained red. Sinusoidal infiltration by the epithelioid tumor cells was highlighted $(\mathrm{a}, \times 100 ; \mathrm{b}, \times 400)$. c The epithelioid tumor cells were weakly positive for Melan-A $(\times 400)$. $d$ The epithelioid tumor cells were negative for $\mathrm{S} 100(\times 400)$. e The epithelioid cells were negative for ALK $(\times 400)$. $\mathrm{f}$ Vimentin was positive in the epithelioid cells $(\times 400)$.

Given that the invasive growth pattern is one of the most important histological features differentiating malignant tumors from benign ones, the case of hepatic AML presented here might cause difficulties in diagnosis and raise differential diagnosis of a mimicker of malignancy. The following tumors should be put into consideration: hepatocellular carcinoma (HCC), epithelioid inflammatory myofibroblastic sarcoma (EIMS), epithelioid angiosarcoma, clear cell sarcoma of soft tissue.

HCC is a malignant tumor with hepatocellular differentiation which has different histopathologic appearance. Tumor cells grow in cords of variable thickness that are separated by sinusoid-like blood spaces in trabecular pattern, is the most common feature in well- and moderately differentiated HCCs. This feature could mimic AML in our case. It is reported that CT and MRI was not helpful in distinguishing AML with HCC [13]. In clinical information, the risk factors of HCC contain hepatitis B or C virus infection, higher serum AFP, among others. In immunohistochemical staining, the tumor cells were positive in hepatocyte (Hep Par-1), CD10 and/or AFP. The capillarization demonstrated by CD34 was also contribute to the right diagnose.

The case we report here have epithelioid tumor cells, obviously invasive growth pattern with lymphocytes and plasma cells infiltration, which might attribute to a wrong diagnosis of EIMS. EIMS is a variant of inflammatory myofibroblastic tumor (IMT), which has some malignant characteristics and was mostly occurred in abdominal cavity of adults. EIMS consists of round-to-epithelioid cells with large and bright nuclei, prominent nucleoci and mitosis. Some regions of stroma were loose or myxoid with inflammatory infiltrates. In addition, the presence of the RANBP2-ALK fusion gene is helpful in the diagnose [14]. Futhermore, positive immunostaining for ALK with a pattern of nuclear membrane or perinuclear staining also be of help in making the diagnosis.

As the slit structures were easily seen in our case, epithelioid angiosarcoma was also put into differential diagnosis. Epithelioid angiosarcoma is a variant of angiosarcoma which has epithelioid endothelial cells, with large vacuolar nucleus, and variable number of mitoses. Irregular vascular lumens can be seen in most tumors, solid flake arrangement was also exsit [15]. Immunohistochemical staining was helpful, because vascular markers such as ERG, CD31, and CD34 could be strongly and diffusely positive in tumor cells.

Finally, we need to exclude the diagnosis of clear cell sarcoma of soft tissue. Clear cell sarcoma of soft tissue could also be positive for HMB45 and other melanoma antigens. However, it usually had larger, plemorphic nucleus with prominent nucleolus [16]. Besides, fat cells and thick-walled blood vessels were absent in morphological evaluation. In addition, melanin immunostains might be positive in $50 \%$ of the cases.

In conclusion, the reported hepatic AML case revealed a relatively rare growth pattern, namely prominent invasive growth pattern. This rare growth pattern was an unusual pathologic appearance in hepatic AML which might promote misdiagnosis. The pathologist should pay attention to this rare morphological feature so as to avoid misdiagnosis. Combined morphology and immunohistochemical staining can lead to correct diagnosis. Although most of hepatic AMLs with invasive growth were biologically benign, long time follow-up is still recommended as the uncertain malignant potential.

\section{References}

1. Bonetti F, Pea M, Martignoni G, Zamboni G (1992) PEC and sugar. Am J Surg Pathol 16: 307-308. [Crossref]

2. Martignoni G, Pea M, Reghellin D, Zamboni G, Bonetti F, et al. (2008) PEComas: the past, the present and the future. Virchows Arch 452: 119-132. [Crossref]

3. Tsui WMS, Colombari R, Portmann BC, Bonetti, F, Thung SN, et al. (1999) Hepatic angiomyolipoma: a clinicopathologic study of 30 cases and delineation of unusual morphologic variants. Am J Surg Pathol 23: 34-48.

4. Nonomura A, Enomoto Y, Takeda M, Tamura T, Kasai T, et al. (2006) Invasive growth of hepatic angiomyolipoma; a hitherto unreported ominous histological feature. Histopathology. 48: 831-835.

5. Nguyen TT, Gorman B, Shields D, Goodman Z (2008) Malignant hepatic angiomyolipoma: report of a case and review of literature. Am J Surg Pathol 32: 793-798.

6. Fukuda Y, Omiya H, Takami K, Mori K, Kodama Y, et al. (2016) Malignant hepatic epithelioid angiomyolipoma with recurrence in the lung 7 years after hepatectomy: a case report and literature review. Surg Case Rep 2: 31. [Crossref]

7. Parfitt JR, Bella AJ, Izawa JI, Wehrli BM (2006) Malignant neoplasm of perivascular epithelioid cells of the liver. Arch Pathol Lab Med 130: 1219-1222.

8. Dalle I, Sciot, De Vos, Aerts, Van Damme, et al. (2000) Malignant angiomyolipoma of the liver: a hitherto unreported variant. Histopathology 36: 443-450.

9. Deng YF, Lin Q, Zhang SH, Ling YM, He JK, et al. (2008) Malignant angiomyolipoma in the liver: a case report with pathological and molecular analysis. Pathol Res Pract 204: $911-918$

10. Folpe AL (2005) Perivascular epithelioid cell neoplasms of soft tissue and gynecologic origin: a clinicopathologic study of 26 cases and review of the literature. Am J Surg Pathol 29: 1558-1575.

11. Bleeker JS, Quevedo JF, Folpe AL (2012) "Malignant" perivascular epithelioid cell neoplasm: risk stratification and treatment strategies. Sarcoma, 2012: 541626.

12. Nese N, Martignoni G, Fletcher CD, Amin MB (2011) Pure epithelioid PEComas (so-called epithelioid angiomyolipoma) of the kidney: A clinicopathologic study of 41 cases: detailed assessment of morphology and risk stratification. Am J Surg Pathol 35: 161-176.

13. Chang ZG, Zhang JM, Ying JQ, Ge YP (2011) Characteristics and treatment strategy of hepatic angiomyolipoma: a series of 94 patients collected from four institutions. $J$ Gastrointestin Liver Dis 20: 65-69.

14. Hallin M, Thway K (2018) Epithelioid Inflammatory Myofibroblastic Sarcoma. Int J Surg Pathol

15. Fletcher CD (1991) Epithelioid angiosarcoma of deep soft tissue: a distinctive tumor readily mistaken for an epithelial neoplasm. Am J Surg Pathol 15: 915-924.

16. Segawa K, Sugita S, Aoyama T, Kubo T, Asanuma H, et al. (2018) Detection of specific gene rearrangements by fluorescence in situ hybridization in 16 cases of clear cell sarcoma of soft tissue and 6 cases of clear cell sarcoma-like gastrointestinal tumor Diagn Pathol 13: 73.

Copyright: (C2018 Zhao C. This is an open-access article distributed under the terms of the Creative Commons Attribution License, which permits unrestricted use, distribution, and reproduction in any medium, provided the original author and source are credited. 